\title{
Isomorphisms of Maximal Subsemigroups of D-classes of Finite Full Transformation Semigroup
}

\author{
Xiang $\mathrm{Li}^{*}$ \\ School of Mathematics, Zunyi Normal College, Zunyi Guizhou 563002, People’s Republic of China \\ ${ }^{*}$ Corresponding author
}

\begin{abstract}
In this paper, by using Green's equivalences, we obtaine isomorphisms of the maximal subsemigroups of $G$ reen-classes( $R$-classes, $L$-classes and $D$-classes).

Keywords_green-classes; maximal subsemigroups; isomorphism
\end{abstract}

\section{INTRODUCTION AND MAIN RESULT}

Let $T_{n}$ be the finite full transformation semigroup on the set $X_{n}=\{1,2, \cdots, n\}(n \geq 3)$, from the Gomes and Howie[1], the Green equicvalences in $T_{n}$ can be characterized as:

$$
\begin{gathered}
\alpha L \beta \Leftrightarrow \operatorname{im} \alpha=\operatorname{im} \beta ; \\
\alpha R \beta \Leftrightarrow \operatorname{ker} \alpha=\operatorname{ker} \beta ; \\
\alpha H \beta \Leftrightarrow i m \alpha=\operatorname{im} \beta, \operatorname{ker} \alpha=\operatorname{ker} \beta,
\end{gathered}
$$

where $\alpha, \beta \in D_{r}$, it is well known that $T_{n}$ has exactly $n$ $D$-classes. The $D$-classes of $T_{n}$ have form $D_{r}=\left\{\alpha \in T_{n}:|i m \alpha|=r\right\}$, for $1 \leq r \leq n$. Especially, when $r=n, D_{r}$ denotes as a symmetric group on $X_{n}$. That is to say, $D_{r}$ consists of all bijections on $X_{n}$.

If $\phi$ is a mapping from a semigroup $S$ into a semigroup $T$, we say that $\phi$ is a homomorphism if $\forall x, y \in S, \phi(x) \phi(y)=\phi(x y)$. And if $\phi$ is both one-one and onto,we shall call it an isomorphism.

In recent years, many scholars have done lots of research deeply on full transformation semigroups and their subsemigroups[2-7]. Especially, In 1998 chein.B. M. and Teclezghi. B. in [2] have studied endomorphisms of finite full transformation semigroups. Tang and Yang in [3,4] have described homomorphisms of two finite full transformation semigroups. But isomorphism of transfor-mation semigroups has been studied rarely. We have obtained the structure of the maximal subsemigroups of $G r e e n$-classes( $R$-classes, $L$-classes and $D$-classes) of $T_{n}$, and showed the maximal subsemigroups of $R$-classes and $L$-classes are also the maximal subsemigroups of $D$-classes(see[5,6]). In this paper, we consider the isomorphism of maximal subsemigroups of $D$-classes.
The main result can be described as follows:

Theorem 1.1. Let $S, T$ be arbitrary maximal subsemigroups of $R$-classes in $D_{r}(2 \leq r \leq n-1)$. If $|E(S)|=|E(T)|$ (denote $E(S)$ the set of all idempotents of $S$ ), then $S \cong T$.

Theorem 1.2. Let $S, T$ be arbitrary maximal subsemigroups of $L$-classes in $D_{r}(2 \leq r \leq n-1)$. If $|E(S)|=|E(T)|$, then $S \cong T$.

Theorem 1.3. Let $S, T$ be arbitrary maximal subsemigroups in the same $D$-class. For each $\alpha \in S, \beta \in T$, we have

$$
S \cong T \Leftrightarrow\left|E\left(R_{\alpha} \cap S\right)\right|=\left|E\left(R_{\beta} \cap T\right)\right|,\left|E\left(L_{\alpha} \cap S\right)\right|=\left|E\left(L_{\beta} \cap T\right)\right| .
$$

Denote $R_{\alpha}$ the $R$-class containing the element $\alpha$.

This paper is organized as follow: in Section 2, we give some lemmas which will be used to prove out main result, and the proof is given in Section 3.

\section{SOME PRELIMINARY RESULTS}

In the section, we give some lemmas in preparation for the proof of our main result. Definitions and terms that are not defined in this article see[1].

Lemma 2.1. Let $a$ be an element of a regular $D$-class $D$ in a semigroup $S$.

(1)If $a^{\prime} \in V(a)$ (the set of inverse of $a$ ), then $a^{\prime} \in D$ and the two $H$-classes $R_{a} \cap L_{a^{\prime}}, L_{a} \cap R_{a^{\prime}}$ contain, respectively, the idempotent $a a^{\prime}$ and $a^{\prime} a$.

(2)If $b$ in $D$ is such that $R_{a} \cap L_{b}$ and $L_{a} \cap R_{b}$ contain idempotents $e, f$, respectively, then $H_{b}$ contains an inverse $a^{*}$ of a such that $a a^{*}=e, a^{*} a=f$.

(3)No $H$-class contains more than one inverse of $a$.

Proof. See [1].

The following result usually known as Green's Lemma: 
Lemma 2.2. ${ }^{[1]}$ Let $a, b$ be $R$-equivalent elements in a semigroups, and let $s, s^{\prime}$ in $S^{1}(S \cup\{1\})$ be such that

$$
a s=b, b s^{\prime}=a \text {. }
$$

Then the right translations $\rho_{s}\left|L_{a}, \rho_{s^{\prime}}\right| L_{b}$ are mutually inverse $R$-class preserving bijections from $L_{a}$ onto $L_{b}$ and $L_{b}$ onto $L_{a}$, respectively.

Lemma 2.3. ${ }^{[1]}$ Let $a, b$ be $L$-equivalent elements in a semigroups, and let $t, t^{\prime}$ in $S^{1}$ be such that

$$
a t=b, b t^{\prime}=a
$$

Then the left translations $\lambda_{t}\left|R_{a}, \lambda_{t^{\prime}}\right| R_{b}$ are mutually inverse $L$-class preserving bijections from $R_{a}$ onto $R_{b}$ and $R_{b}$ onto $R_{a}$, respectively.

By Lemma 2.2 and 2.3, let $H=H_{e}$ and $K=H_{f}$ are two $H$-classes in the same $R$-class, where $e \in E(H)$, $f \in E(K)$, then $\rho_{f}$ is an isomorphism from $H_{e}$ onto $H_{f}$, with inverse $\rho_{e}$ from $H_{f}$ onto $H_{e}$. Similarly, if $H=H_{g}$ and $K=H_{h}$ are two $H$-classes in the same $L$-class, where $g \in E(H), h \in E(K)$, then $\lambda_{g}$ is an isomorphism from $H_{h}$ onto $H_{g}$, with inverse $\lambda_{h}$ from $H_{g}$, onto $H_{h}$.

Lemma 2.4. Let $S, T$ be arbitrary finite semigroups. If $S \cong T$, then $|E(S)|=|E(T)|$.

Proof. If $\varphi$ be isomorphism from $S$ onto $T$, then $\varphi(E(S)) \subseteq E(T)$, since $S, T$ be finite semigroups, we have $|E(S)|=|\varphi(E(S))| \leq|E(T)|$. Similarly, we have $|E(T)| \leq|E(S)|$, further $|E(T)|=|E(S)|$.

Lemma 2.5. Let $S, T$ be arbitrary semigroups, a map $\varphi: S \rightarrow T$ be semigroup morphism. Then we have

$$
\begin{gathered}
a R b \Leftrightarrow \varphi(a) R \varphi(b), \forall a, b \in S ; \\
a L b \Leftrightarrow \varphi(a) L \varphi(b), \forall a, b \in S ; \\
e \in E(S) \Rightarrow \varphi(e) \in E(S) .
\end{gathered}
$$

Proof. (1)Suppose $a R b$, there exist $u, v \in S$ such that $a=b u, b=a v$, then

$$
\varphi(a)=\varphi(b u)=\varphi(b) \varphi(u), \varphi(b)=\varphi(a v)=\varphi(a) \varphi(v)
$$

Therefore $\varphi(a) R \varphi(b)$.

(2)Similar to (1);

(3)Let $e \in E(S)$, then $\varphi(e)=\varphi(e e)=\varphi(e) \varphi(e)$, thus $\varphi(e) \in E(S)$.

Therefore, one of the necessary conditions about isomorphism of maximal subsemigroups of $D_{r}$ is that the number of idempotents is equal.

\section{PROOF OF THEOREM}

In this section, we give the proof of Theorem 1.1,1.2 and 1.3 , and study the isomorphism of maximal subsemigroups of $R$-classes, $L$-classes and $D$-classes.

Proof of Theorem 1.1. Let

$$
S=\bigcup_{i=1}^{r} H_{e_{i}}, T=\bigcup_{i=1}^{r} H_{f_{i}}(2 \leq r \leq n-1),
$$

where $e_{i} \in E(S), f_{i} \in E(T)$. Let $\varphi_{1}: H_{e_{1}} \rightarrow H_{f_{1}}$,

$x \rightarrow a^{\prime} x a$. By Lemma 3.1, there exist $a \in R_{e_{1}} \cap L_{f_{1}}$,

$a^{\prime} \in L_{e_{1}} \cap R_{f_{1}}$ such that $a a^{\prime}=e_{1}, a^{\prime} a=f_{1}$. Then we have that $\varphi_{1}$ is an isomorphism from $H_{e_{1}}$ onto $H_{f_{1}}$.

Let

$$
\rho_{e_{i}}: H_{e_{1}} \rightarrow H_{e_{i}}, x \rightarrow x e_{i}, \rho_{f_{i}}: H_{f_{1}} \rightarrow H_{f_{i}}, x \rightarrow x f_{i}
$$

Then $\rho_{e_{i}}, \rho_{f_{i}}$ are isomorphic from $H_{e_{1}}$ onto $H_{e_{i}}$ and $H_{f_{1}}$ onto $H_{f_{i}}$, respectively.

Thus the map $\varphi_{i}=\rho^{-1} \varphi_{1} \rho_{f_{i}}(i \neq 1)$ is an isomorphism from $H_{e_{i}}$ onto $H_{f_{i}}$.

Give a function from $S$ to $T$

$$
g(x)=\left\{\begin{array}{l}
\varphi_{1}(x) \quad x \in H_{e_{1}} \\
\varphi_{i}(x) \quad x \in H_{e_{i}}(i \neq 1)
\end{array} .\right.
$$

It's easy to see that $g$ is a bijection.

For arbitrary $x_{1} \in H_{e_{1}}, x_{2} \in H_{e_{i}}(i \neq 1)$, we have 


$$
\begin{gathered}
g\left(x_{1} x_{2}\right)=\varphi_{i}\left(x_{1} x_{2}\right)=\left(x_{1} x_{2}\right) \rho_{e_{i}}^{-1} \varphi_{1} \rho_{f_{i}}=a^{\prime} x_{1} x_{2} e_{1} a f_{i}=a^{\prime} x_{1} x_{2} a f_{i}, \\
g\left(x_{1}\right) g\left(x_{2}\right)=\varphi_{1}\left(x_{1}\right) \varphi_{i}\left(x_{2}\right)=a^{\prime} x_{1} a a^{\prime} x_{2} e_{1} a f_{i} \\
=a^{\prime} x_{1} e_{1} x_{2} a f_{i}=a^{\prime} x_{1} x_{2} a f_{i} .
\end{gathered}
$$

Thus $g\left(x_{1} x_{2}\right)=g\left(x_{1}\right) g\left(x_{2}\right)$, similarly, we have

$$
g\left(x_{2} x_{1}\right)=g\left(x_{2}\right) g\left(x_{1}\right)
$$

For arbitrary $x_{1} \in H_{e_{i}}, x_{2} \in H_{e_{i}}(i \neq j, i, j \neq 1)$,

we have

$$
\begin{gathered}
g\left(x_{1} x_{2}\right)=\varphi_{j}\left(x_{1} x_{2}\right)=\left(x_{1} x_{2}\right) \rho_{e_{j}}^{-1} \varphi_{1} \rho_{f_{j}} \\
=a^{\prime} x_{1} x_{2} e_{1} a f_{j}=a^{\prime} x_{1} x_{2} a f_{j}, \\
g\left(x_{1}\right) g\left(x_{2}\right)=\left(x_{1} \rho_{e_{i}}^{-1} \varphi_{1} \rho_{f_{i}}\right)\left(x_{2} \rho_{e_{j}}^{-1} \varphi_{1} \rho_{f_{j}}\right) \\
=a^{\prime} x_{1} e_{1} a f_{i} a x_{2} e_{1} a f_{j}=a^{\prime} x_{1} a f_{i} a^{\prime} x_{2} a f_{j} \\
=a^{\prime} x_{1} a a^{\prime} x_{2} a f_{j}=a^{\prime} x_{1} x_{2} a f_{j} .
\end{gathered}
$$

Thus $g\left(x_{1} x_{2}\right)=g\left(x_{1}\right) g\left(x_{2}\right)$.

Therefore, $g$ is an isomorphism from $S$ onto $T$.

The proof of Theorem 1.2 is similarly to Theorem 1.1 .

Proof of Theorem 1.3. Firstly, we shall prove the sufficient condition.

Since the structural of $S, T$ and for arbitrary $e \in E(S)$, $f \in E(T)$, we have

$$
\left|E\left(L_{e} \cap S\right)\right|=\left|E\left(L_{f} \cap T\right)\right|,\left|E\left(R_{e} \cap S\right)\right|=\left|E\left(R_{f} \cap T\right)\right| .
$$

Then the arrangement of idempotents of $S$ and $T$ can be seen as two matrix with equal rank, we denote them by $A_{k l}$ and $B_{k l}$, where $e_{i j}, f_{i j}$ standing the $i$ row and $j$ column in $A_{k l}$ and $B_{k l}$ respectively, and

$$
e_{i j} \in E\left(A_{k l}\right), f_{i j} \in E\left(B_{k l}\right) \text {. }
$$

Similar to Theorem 1.1, define an isomorphism

$$
\varphi_{i j}: H_{e_{i j}} \rightarrow H_{f_{i j}} .
$$

If $i=1, j=1$, $\varphi_{11}(x)=a^{\prime} x a$,

where

$$
a \in R_{e_{11}} \cap L_{f_{11}}, a^{\prime} \in R_{f_{11}} \cap L_{e_{11}}, a a^{\prime}=e_{11}, a^{\prime} a=f_{11},
$$

it's easy to see by Lemma 2.1 .

If $i=1, j \neq 1$,

$$
\varphi_{1 j}=\rho_{e_{1 j}}^{-1} \varphi_{11} \rho_{f_{1 j}},
$$

where $\rho_{e_{1 j}}^{-1}=\rho_{e_{11}}$.

If $i \neq 1, j=1$,

$$
\varphi_{i 1}=\lambda_{e_{i 1}}^{-1} \varphi_{11} \lambda_{f_{i 1}}
$$

where $\lambda_{e_{i 1}}^{-1}=\lambda_{e_{11}}$.

If $i \neq 1, j \neq 1$,

$$
\varphi_{i j}=g_{1}^{-1} \varphi_{11} g_{2} \text {, }
$$

where

$g_{1}: H_{e_{11}} \rightarrow H_{e_{i j}}, x \rightarrow x \rho_{e_{1 j}} \lambda_{e_{i j}}, g_{2}: H_{f_{11}} \rightarrow H_{f_{i j}}, x \rightarrow x \rho_{f_{1 j}} \lambda_{f_{i j}}$

Thus

$$
\varphi_{i j}=\rho_{e_{i 1}} \lambda_{e_{11}} \varphi_{11} \lambda_{f_{i 1}} \rho_{f_{i j}}
$$

Further, we can give a map $g: S \rightarrow T:$

$$
g(x)= \begin{cases}a^{\prime} x a, & x \in H_{e_{11}}, \\ \varphi_{i j}(x), & x \in H_{e_{i j}} .\end{cases}
$$

It is easy to show that $g$ is a bijection from $S$ onto $T$. Next, we distinguish 6 cases:

Case 1. For arbitrary

$$
x_{1} \in H_{e_{11}}, x_{2} \in H_{e_{1 j}}(j \neq 1)
$$

then

$$
g\left(x_{1} x_{2}\right)=g\left(x_{1}\right) g\left(x_{2}\right), g\left(x_{2} x_{1}\right)=g\left(x_{2}\right) g\left(x_{1}\right)
$$

by Theorem 1.1 .

For arbitrary 


$$
x_{1} \in H_{e_{11}}, x_{2} \in H_{e_{11}}(i \neq 1)
$$

similarly, we have

$$
g\left(x_{1} x_{2}\right)=g\left(x_{1}\right) g\left(x_{2}\right), g\left(x_{2} x_{1}\right)=g\left(x_{2}\right) g\left(x_{1}\right) .
$$

Case 2. For arbitrary

$$
x_{1} \in H_{e_{11}}, x_{2} \in H_{e_{i j}}(i, j \neq 1),
$$

then

$$
\begin{aligned}
& g\left(x_{1} x_{2}\right)=\varphi_{1 j}\left(x_{1} x_{2}\right)=a^{\prime} x_{1} x_{2} e_{11} a f_{1 j}=a^{\prime} x_{1} x_{2} a f_{1 j}, \\
& \begin{aligned}
g\left(x_{1}\right) g\left(x_{2}\right) & =a^{\prime} x_{1} a f_{i 1} a^{\prime} e_{11} x_{2} e_{i 1} a f_{i j} \\
& =a^{\prime} x_{1} a a^{\prime} e_{11} x_{2} e_{i j} e_{11} a f_{i 1} f_{1 j} \\
& =a^{\prime} x_{1}\left(x_{2} e_{i j}\right)\left(e_{11} a f_{i 1}\right) f_{1 j} \\
& =a^{\prime} x_{1} x_{2} a f_{1 j} .
\end{aligned}
\end{aligned}
$$

Thus, $g\left(x_{1} x_{2}\right)=g\left(x_{1}\right) g\left(x_{2}\right)$. Similarly,

$$
g\left(x_{2} x_{1}\right)=g\left(x_{2}\right) g\left(x_{1}\right) .
$$

Case 3. For arbitrary

$$
x_{1} \in H_{e_{1},}, x_{2} \in H_{e_{11}}(i, j \neq 1)
$$

then

$$
\begin{aligned}
g\left(x_{1} x_{2}\right)= & \varphi_{11}\left(x_{1} x_{2}\right)=a^{\prime} x_{1} x_{2} a, \\
g\left(x_{1}\right) g\left(x_{2}\right) & =a^{\prime} x_{1} e_{11} a f_{1 j} f_{i 1} a^{\prime} e_{11} x_{2} a \\
& =a^{\prime} x_{1} a f_{11} a^{\prime} e_{11} x_{2} a \\
& =a^{\prime} x_{1} e_{11} x_{2} a \\
& =a^{\prime}\left(x_{1} e_{1 j}\right)\left(e_{i 1} x_{2}\right) a \\
& =a^{\prime} x_{1} x_{2} a .
\end{aligned}
$$

Thus, $g\left(x_{1} x_{2}\right)=g\left(x_{1}\right) g\left(x_{2}\right)$. Similarly,

$$
g\left(x_{2} x_{1}\right)=g\left(x_{2}\right) g\left(x_{1}\right) .
$$

Case 4. For arbitrary

$$
x_{1} \in H_{e_{1 m}}, x_{2} \in H_{e_{i j}}(m, i, j \neq 1) \text {, }
$$

then

$$
\begin{aligned}
& g\left(x_{1} x_{2}\right)=\varphi_{1 j}\left(x_{1} x_{2}\right)=a^{\prime} x_{1} x_{2} e_{11} a f_{1 j}=a^{\prime} x_{1} x_{2} a f_{1 j}, \\
& g\left(x_{1}\right) g\left(x_{2}\right)=a^{\prime} x_{1} e_{11} a f_{1 m} f_{i 1} a^{\prime} e_{11} x_{2} e_{i 1} a f_{i j}
\end{aligned}
$$

$$
\begin{aligned}
& =a^{\prime} x_{1} a f_{11} a^{\prime} x_{2} e_{i j} e_{11} a f_{i 1} f_{1 j} \\
& =a^{\prime} x_{1} e_{11} x_{2} e_{i j}\left(e_{11} a\right) f_{1 j} \\
& =a^{\prime}\left(x_{1} e_{1 m}\right)\left(e_{i 1} x_{2} e_{i j}\right) a f_{1 j}
\end{aligned}
$$

$=a^{\prime} x_{1} x_{2} a f_{1 j}$.

Thus, $g\left(x_{1} x_{2}\right)=g\left(x_{1}\right) g\left(x_{2}\right)$. Similarly,

Case 5. For arbitrary

$$
g\left(x_{2} x_{1}\right)=g\left(x_{2}\right) g\left(x_{1}\right) .
$$

$$
x_{1} \in H_{e_{n 1}}, x_{2} \in H_{e_{i j}}(n, i, j \neq 1),
$$

then

$$
\begin{aligned}
& g\left(x_{1} x_{2}\right)=\varphi_{n j}\left(x_{1} x_{2}\right)=f_{n 1} a^{\prime} e_{11} x_{1} x_{2} e_{n 1} a f_{n j}=f_{n 1} a^{\prime} x_{1} x_{2} e_{n j} e_{11} a f_{n j} \\
& =f_{n 1} a^{\prime} x_{1} x_{2} a f_{n 1} f_{1 j}=f_{n 1} a^{\prime} x_{1} x_{2} a f_{1 j}, \\
& g\left(x_{1}\right) g\left(x_{2}\right)=f_{n 1} a^{\prime} e_{11} x_{1} a f_{i 1} a^{\prime} e_{11} x_{2} e_{i 1} a f_{i j} \\
& =f_{n 1} a^{\prime} x_{1} a a^{\prime}\left(x_{2} e_{i j}\right)\left(e_{11} a f_{n 1}\right) f_{1 j}=f_{n 1} a^{\prime} x_{1} x_{2} f_{1 j} .
\end{aligned}
$$

Thus, $g\left(x_{1} x_{2}\right)=g\left(x_{1}\right) g\left(x_{2}\right)$. Similarly,

$$
g\left(x_{2} x_{1}\right)=g\left(x_{2}\right) g\left(x_{1}\right) .
$$

Case 6. For arbitrary

$$
x_{1} \in H_{e_{i j}}, x_{2} \in H_{e_{m n}}(i, j, m, n \neq 1),
$$

then

$$
\begin{gathered}
g\left(x_{1} x_{2}\right)=\varphi_{i n}\left(x_{1} x_{2}\right)=f_{i 1} a^{\prime} e_{i 1} x_{1} x_{2} e_{i 1} a f_{i n} \\
=f_{i 1} a^{\prime}\left(x_{1} x_{2}\right) e_{i n}\left(e_{11} a f_{i 1}\right) f_{1 n}=f_{i 1} a^{\prime} x_{1} x_{2} a f_{1 n}, \\
g\left(x_{1}\right) g\left(x_{2}\right)=f_{i 1} a^{\prime} e_{11} x_{1} e_{i 1} a f_{i j} f_{m 1} a^{\prime} e_{11} x_{2} e_{m 1} a f_{m n} \\
=f_{i 1} a^{\prime}\left(x_{1} e_{i j}\right)\left(e_{11} a\right) f_{i j} f_{m 1} a^{\prime}\left(x_{2} e_{m n}\right)\left(e_{11} a\right) f_{m n} \\
=f_{i 1} a^{\prime} x_{1}\left(a f_{i 1}\right) a^{\prime} x_{2}\left(a f_{m 1}\right) f_{1 n}=f_{i 1} a^{\prime} x_{1} e_{11} x_{2} a f_{1 n} \\
=f_{i 1} a^{\prime}\left(x_{1} e_{1 j}\right)\left(e_{m 1} x_{2}\right) a f_{1 n}=f_{i 1} a^{\prime} x_{1} x_{2} a f_{1 n} .
\end{gathered}
$$

Thus,

$$
g\left(x_{1} x_{2}\right)=g\left(x_{1}\right) g\left(x_{2}\right) .
$$

From the discussion above, we have shown that $g$ is an isomorphism from $S$ onto $T$, thus we have shown the sufficient condition.

Next, we shall show the necessary condition. Let $\varphi$ be a semigroup isomorphism from $S$ onto $T$. Then for arbitrary $e \in E(S)$, by Lemma 2.5 we have

$\left|E\left(R_{e} \cap S\right)\right| \leq\left|E\left(R_{\varphi(e)} \cap T\right)\right|,\left|E\left(L_{e} \cap S\right)\right| \leq\left|E\left(L_{\varphi(e)} \cap T\right)\right|$.

For arbitrary $\alpha \in S, \beta \in T$, by Lemma 2.4 we deduce

$\left|E\left(R_{e} \cap S\right)\right|=\left|E\left(R_{\alpha} \cap S\right)\right|,\left|E\left(L_{e} \cap S\right)\right|=\left|E\left(L_{\alpha} \cap S\right)\right|$, 
$\left|E\left(R_{\varphi(e)} \cap T\right)\right|=\left|E\left(R_{\beta} \cap T\right)\right|,\left|E\left(L_{\varphi(e)} \cap T\right)\right|=\left|E\left(L_{\beta} \cap T\right)\right|$.

Further,

$\left|E\left(R_{\alpha} \cap S\right)\right| \leq\left|E\left(R_{\beta} \cap T\right)\right|,\left|E\left(L_{\alpha} \cap S\right)\right| \leq\left|E\left(L_{\beta} \cap T\right)\right|$.

Notice that $\varphi^{-1}$ is an isomorphism from $T$ onto $S$, thus in the same way we have

$\left|E\left(R_{\alpha} \cap S\right)\right| \geq\left|E\left(R_{\beta} \cap T\right)\right|,\left|E\left(L_{\alpha} \cap S\right)\right| \geq\left|E\left(L_{\beta} \cap T\right)\right|$,

for arbitrary $\alpha \in S, \beta \in T$.

Hence,

$\left|E\left(R_{\alpha} \cap S\right)\right|=\left|E\left(R_{\beta} \cap T\right)\right|,\left|E\left(L_{\alpha} \cap S\right)\right|=\left|E\left(L_{\beta} \cap T\right)\right|$.

We have shown the necessary condition.

From the discussion above, the proof of Proposition 1.3 is complete.

\section{ACKNOWLEDGMENT}

This article is supported by the Science and Technology Foundation of Guizhou Province( No.LH[2015]7047 ).

\section{REFERENCES}

[1] J. M. Howie, Fundamentals of Semigroup of Theory, Oxford Press, London , 1995.

[2] B. M. Schein, B. Teclezghi, "Endomorphisms of finite full transformation semigroups,” Proceedings of the American Mathematical Society, vol. 126, pp. 2579-2587, 1998.

[3] H. Tang, X. Yang, "Homomorphisms of Two Finite Transformation Semigroups I," Journal of Huangzhou Normal University(Natural Science Edition), vol. 14, pp. 527-530, Sep. 2015.

[4] H. Tang, X. Yang, "Homomorphisms of Two Finite Full Transformation Semigroups II," Journal of Huangzhou Normal University(Natural Science Edition), vol. 15, pp. 178-183 , Mar. 2016.

[5] X. Li, L. Deng, H. Zhang, J. Ouyang, "Maximal subsemigroups of some special subsets of $D$-classes $D$ of Finite Full Transformation Semigroup $T_{n}$," Journal of Guizhou Normal University(Natural Science), vol 26, pp. 88-89, Feb. 2008

[6] X. Li, H. Huang, "Maximal subsemigroups of $D$-classes for finite full transformation semigroup,"Journal of Nanchang University(Natural Science), vol. 38, pp. 524-526, Dec. 2014.

[7] H. Tang, Z. Yi, P. Deng, "Structure about Connected or Irreducible Automorphism Group of Transformation Semigroup,” Guangxi Sciences, vol. 15, pp. 338-340, Nov. 2008. 